\title{
(2) OPEN ACCESS \\ Predicting the immediate impact of national lockdown on neovascular age-related macular degeneration and associated visual morbidity: an INSIGHT Health Data Research Hub for Eye Health report
}

\author{
Susan P Mollan, ${ }^{1}$ Dun Jack Fu $\left(10,{ }^{2}\right.$ Ching-Yi Chuo, ${ }^{3}$ Jacqueline G Gannon, ${ }^{3}$ \\ Wen Hwa Lee, ${ }^{4} \mathrm{~J}$ Jill Hopkins, ${ }^{3}$ Cian Hughes, ${ }^{5}$ Alastair K Denniston $\left(10,{ }^{6}\right.$ \\ Pearse A Keane, ${ }^{7}$ Ronald Cantrell ${ }^{3}$
}

- Additional supplemental material is published online only. To view, please visit the journal online (http://dx.doi. org/10.1136/bjophthalmol2021-319383).

1 Ophthalmology, University Hospitals Birmingham, Birmingham, UK

${ }^{2}$ Research Centre, Moorfields Eye Hospital NHS Foundation Trust, London, UK ${ }^{3}$ Genentech Inc, South San Francisco, California, USA ${ }^{4}$ Action Against Age-Related Macular Degeneration, Oxford, UK

${ }^{5}$ Google Inc, Mountain View, California, USA

${ }^{6}$ Department of Ophthalmology, University Hospitals Birmingham NHSFT, Birmingham, UK

${ }^{7}$ Medical Retina, Moorfields Eye Hospital NHS Foundation Trust, London, UK

\section{Correspondence to}

Dr Susan P Mollan,

Ophthalmology, University Hospitals Birmingham, Birmingham, B15 2TH, UK; soozmollan@doctors.org.uk

Received 6 April 2021 Accepted 30 August 2021

Check for updates

(c) Author(s) (or their employer(s)) 2021. Re-use permitted under CC BY-NC. No commercial re-use. See rights and permissions. Published by BMJ.

To cite: Mollan SP, Fu DJ, Chuo C-Y, et al.

Br J Ophthalmol Epub ahead of print: [please include Day Month Year]. doi:10.1136/ bjophthalmol-2021-319383

\begin{abstract}
Objective Predicting the impact of neovascular agerelated macular degeneration (nAMD) service disruption on visual outcomes following national lockdown in the UK to contain SARS-CoV-2.

Methods and analysis This retrospective cohort study includes deidentified data from 2229 UK patients from the INSIGHT Health Data Research digital hub. We forecasted the number of treatment-naïve nAMD patients requiring anti-vascular endothelial growth factor (anti-VEGF) initiation during UK lockdown (16 March 2020 through 31 July 2020) at Moorfields Eye Hospital (MEH) and University Hospitals Birmingham (UHB). Best-measured visual acuity (VA) changes without antiVEGF therapy were predicted using post hoc analysis of Minimally Classic/Occult Trial of the Anti-VEGF Antibody Ranibizumab in the Treatment of Neovascular AMD trial sham-control arm data $(\mathrm{n}=238)$.

Results At our centres, 376 patients were predicted to require anti-VEGF initiation during lockdown (MEH: 325; UHB: 51). Without treatment, mean VA was projected to decline after 12 months. The proportion of eyes in the MEH cohort predicted to maintain the key positive visual outcome of $\geq 70$ ETDRS letters (Snellen equivalent 6/12) fell from $25.5 \%$ at baseline to $5.8 \%$ at 12 months (UHB: 9.8\%-7.8\%). Similarly, eyes with VA <25 ETDRS letters (6/96) were predicted to increase from $4.3 \%$ to $14.2 \%$ at MEH (UHB: $5.9 \%-7.8 \%$ ) after 12 months without treatment.
\end{abstract}

Conclusions Here, we demonstrate how combining data from a recently founded national digital health data repository with historical industry-funded clinical trial data can enhance predictive modelling in nAMD. The demonstrated detrimental effects of prolonged treatment delay should incentivise healthcare providers to support nAMD patients accessing care in safe environments. Trial registration number NCT00056836.

\section{INTRODUCTION}

Neovascular age-related macular degeneration (nAMD) is the leading cause of irreversible blindness and vision impairment in the elderly population of high-income countries such as the UK. ${ }^{12}$ Visual impairment caused by nAMD can

\section{Key messages}

What is already known about this subject?

- Enforced national lockdowns to limit the spread of SARS-CoV-2 across the world have led to some restrictions in accessing non-essential primary care and hospital services.

- Delays in neovascular age-related macular degeneration (nAMD) diagnosis and treatment were subsequently reported, but the impact of disruption in ophthalmic services on treatmentnaïve patients with nAMD requiring antivascular endothelial growth factor therapy is unknown.

What are the new findings?

- In this retrospective cohort study, using models based on data from the INSIGHT Health Data Research digital hub and the landmark Minimally Classic/Occult Trial of the Anti-VEGF Antibody Ranibizumab in the Treatment of Neovascular AMD trial, 376 nAMD patients were forecasted to have required treatment initiation at two major clinical centres during the UK national lockdown.

- Delays in their treatment were projected to have clinically relevant effects on their visual acuity.

How might these results change the focus of research or clinical practice?

- Efficient resumption of ophthalmic services following national lockdown is crucial to maximise positive visual outcomes for nAMD patients.

lead to devastating decrements in patients' quality of life and poses a growing healthcare challenge, with the number of patients affected estimated to increase by $>30 \%$ in a decade. ${ }^{3-7}$ Critically, visual prognosis can be substantially improved with intravitreal injection of vascular endothelial growth factors inhibitors (anti-VEGF). ${ }^{89}$ It has been demonstrated that timely treatment initiation and active 
surveillance are essential for preservation of vision in patients with nAMD. ${ }^{10}$

The emergence and rapid spread of the new and highly contagious SARS-CoV-2, the pathogen causing COVID-19, has challenged healthcare systems across the globe. The exponential rise in severe COVID-19 cases has mandated a pandemic response requiring a redirection of healthcare resources to intensive care units, and measures to protect uninfected patients and healthcare providers from infection. These efforts have effectively shut down health services that are not critical for life. During the first enforced UK national lockdown, access to primary care services, optometric and hospital eye services was strictly limited to absolute emergencies. This led to delay of new-onset nAMD diagnosis and continuation of care for those already on the nAMD treatment pathway. ${ }^{11-13}$

The impact of this disruption on ophthalmic services and consequently on visual outcomes remains undetermined. ${ }^{14}$ Accurate forecasting of the disruption's impact on ophthalmic services may have on nAMD treatment outcomes is important on several levels to maintain a high quality of care. Good predictions are required to inform triage and treatment strategies once services resume. Furthermore, they may support patient-level prognosis and shared decision-making. Lastly, forecasting visual outcomes is relevant in underpinning public health messaging and patient outreach services that ensure all patients are empowered to seek necessary care.

The INSIGHT Health Data Research Hub for Eye Health in the UK is uniquely positioned to explore such national health trends. As a national data collection initiative, INSIGHT aims to maximise the benefits and impact of historical, deidentified, patient-level UK National Health Service (NHS) hospital admission and electronic health record data by establishing formal collaborations between NHS Trusts, academia, patients, charities and industry. To this end, data from University Hospitals Birmingham (UHB) and Moorfields Eye Hospital NHS Foundation Trusts (MEH) were assembled to describe the period trends for patients initiating anti-VEGF therapy for $\mathrm{nAMD}$ and to forecast the number and characteristics of patients needing to initiate anti-VEGF for nAMD during the national lockdown.

To extrapolate the impact of treatment delay, the landmark Minimally Classic/Occult Trial of the Anti-VEGF Antibody Ranibizumab in the Treatment of Neovascular AMD trial (MARINA) ${ }^{8}$ sham-control arm data were used to model visual outcomes of the patients projected to have their treatment delayed by the COVID-19 lockdown. The MARINA trial was a phase III, randomised, multicentre, double-masked, shamcontrolled study enrolling participants diagnosed with minimally classic nAMD or occult with no classic nAMD. Importantly, the MARINA trial is the last published study to include a shamcontrol arm, as anti-VEGF treatment became the gold standard of care for active nAMD thereafter. ${ }^{15}$

\section{METHODS}

\section{Study design}

This retrospective cohort study includes treatment-naïve patients with nAMD initiating anti-VEGF therapy during the pre-COVID-19 era (1 January 2018 through 15 March 2020) at UHB and MEH. These data were used to forecast nAMD patients requiring treatment initiation in a specific time window of national lockdown where non-essential ophthalmic services were interrupted as part of the pandemic response in the UK (16 March 2020 through 31 July 2020). Visual outcomes were modelled based on patients in the control arm of the MARINA trial.
Table 1 Cohort demography and clinical features at baseline

\begin{tabular}{|c|c|c|c|}
\hline & $\begin{array}{l}\text { Overall } \\
(n=2196)\end{array}$ & $\begin{array}{l}\text { MEH } \\
(n=1929)\end{array}$ & $\begin{array}{l}\text { UHB } \\
(n=267)\end{array}$ \\
\hline \multicolumn{4}{|l|}{$A$} \\
\hline \multicolumn{4}{|l|}{$\operatorname{Sex} \mathrm{n}(\%)$} \\
\hline Female & $1350(61.5)$ & $1167(60.5)$ & $183(68.5)$ \\
\hline Male & 846 (38.5) & 762 (39.5) & $84(31.5)$ \\
\hline \multicolumn{4}{|l|}{ Age (years) n (\%) } \\
\hline$<65$ & $133(6.1)$ & $125(6.5)$ & $8(3.0)$ \\
\hline $65-69$ & $154(7.0)$ & $139(7.2)$ & $15(5.6)$ \\
\hline $70-74$ & $312(14.2)$ & $271(14.0)$ & $41(15.4)$ \\
\hline $75-79$ & 407 (18.5) & $369(19.1)$ & $38(14.2)$ \\
\hline $80-84$ & $515(23.5)$ & $447(23.2)$ & $68(25.5)$ \\
\hline $85+$ & $675(30.7)$ & $578(30.0)$ & $97(36.3)$ \\
\hline \multicolumn{4}{|l|}{ Ethnicity n (\%) } \\
\hline White & $1104(50.3)$ & $905(46.9)$ & $199(74.5)$ \\
\hline Not white & $959(43.7)$ & $945(49.0)$ & $14(5.2)$ \\
\hline Not reported & $133(6.1)$ & $79(4.1)$ & $54(20.2)$ \\
\hline \multicolumn{4}{|l|}{ Smoking status $\mathrm{n}(\%)$} \\
\hline Yes & $149(6.8)$ & $140(7.3)$ & $9(3.4)$ \\
\hline \multicolumn{4}{|l|}{ B } \\
\hline \multicolumn{4}{|l|}{$\begin{array}{l}\text { Visual acuity (VA) } \\
\text { ETDRS letters }\end{array}$} \\
\hline Mean (SD) & $55.4(16.8)$ & $55.9(16.3)$ & $51.7(20.0)$ \\
\hline Median (min, max) & $59.0(0,94.0)$ & $60.0(0,90.0)$ & $55.0(0,94.0)$ \\
\hline \multicolumn{4}{|l|}{$V A \geq 70 n(\%)$} \\
\hline Yes & $563(25.6)$ & $503(26.1)$ & $60(22.5)$ \\
\hline No & $1633(74.4)$ & $1426(73.9)$ & 207 (77.5\%) \\
\hline \multicolumn{4}{|l|}{ VA $\leq 20 n(\%)$} \\
\hline Yes & $55(2.5)$ & $40(2.1)$ & $15(5.6)$ \\
\hline No & $2141(97.5)$ & 1889 (97.9) & $252(94.4)$ \\
\hline \multicolumn{4}{|l|}{ VA $<25 n(\%)$} \\
\hline Yes & $139(6.3)$ & $112(5.8)$ & $27(10.1)$ \\
\hline No & 2057 (93.7) & $1817(94.2)$ & $240(89.9)$ \\
\hline No or unknown & 2047 (93.2) & $1789(92.7)$ & $258(96.6)$ \\
\hline
\end{tabular}

Mean, SD and distribution are shown for (A) demography (sex, ethnicity, smoking status and age) and (B) visual parameters in approximate ETDRS letters of patients with nAMD at initiation of anti-VEGF therapy at MEH and UHB.

MEH, Moorfields Eye Hospital; nAMD, neovascular age-related macular degeneration; UHB, University Hospitals Birmingham; VEGF, vascular endothelial growth factor.

\section{Data source}

Electronic health record data gathered as part of routine clinical care at UHB and MEH between 1 January 2018 and 15 March 2020 were obtained via the INSIGHT collaborative Health Data Research Hub for Eye Health—a formal collaboration between UHB, $\mathrm{MEH}$, Roche, Google, Action Against AMD and the University of Birmingham. Demographic characteristics included sex, ethnicity, age at diagnosis and smoking status. To ensure deidentification of patient data, age and ethnicity were categorised. Best-measured visual acuity (VA) with habitual correction or pinhole as part of clinical examination using ETDRS charts was recorded. For any cases where VA was reported in Snellen annotation, approximate ETDRS was extrapolated and taken forward for analysis. ${ }^{16}$

\section{Participants}

Treatment-naïve nAMD patients requiring anti-VEGF therapy initiation at $\mathrm{MEH}$ and UHB between 1 January 2018 and 15 March 2020. Study eye was designated as the first eye diagnosed 


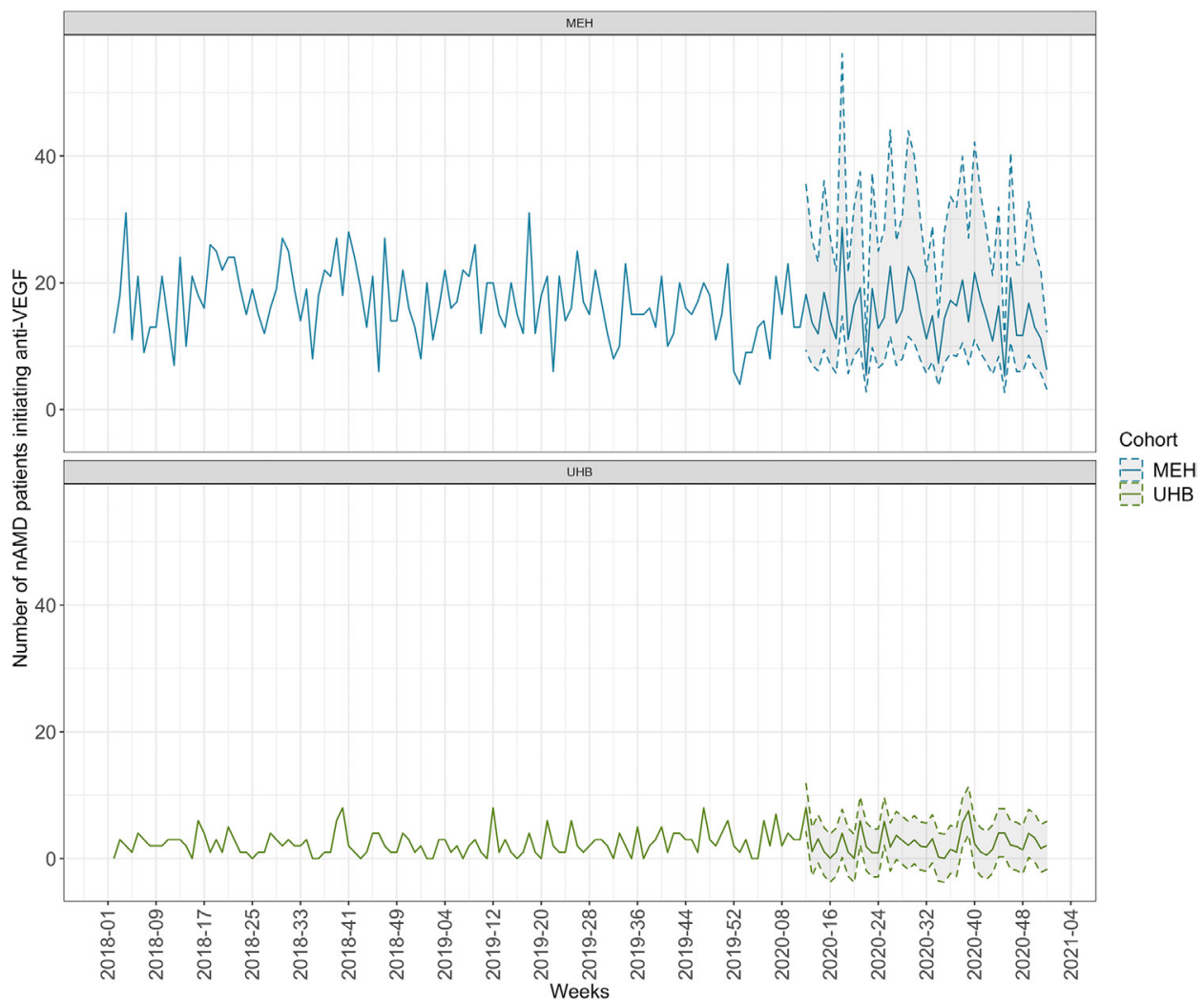

Figure 1 Period trends of anti-vascular endothelial growth factor (anti-VEGF) treatment initiation for neovascular age-related macular degeneration (nAMD). Actual (solid line) and predicted (solid line with CIs) number of patients per week needing to initiate anti-VEGF therapy for nAMD at Moorfields Eye Hospital (MEH) (top panel; blue) and University Hospitals Birmingham (UHB) (bottom panel; green); $95 \%$ (grey) Cl are depicted.

with nAMD. If both eyes presented simultaneously, right eye was designated as study eye.

\section{Outcomes}

The primary outcome was the number of patients forecasted to require initiation of anti-VEGF therapy during the UK national lockdown between 16 March 2020 and 31 July 2020. Predicted visual outcomes if patients were left untreated were also assessed, including: change in VA from baseline; VA of $\geq 70$ ETDRS letters (Snellen equivalent 6/12); VA of $<25$ ETDRS letters (6/96) and VA of $\leq 20$ ETDRS letters (3/60). Each of these end points were considered over 12 months following predicted treatment initiation.

The threshold of 70 ETDRS letters (6/12) is an indicator of patient independence and thus commonly used to signify positive visual outcomes. It is: (1) the International Council of Ophthalmology's threshold for good independent vision ${ }^{17}{ }^{18}$; (2) the legal threshold for driving in the $\mathrm{UK}^{19}$ and (3) the minimum VA required to read small print. ${ }^{20}$ Accordingly, it is recommended as a key threshold by numerous consortia. ${ }^{17} 18$ Conversely, a VA of $<25$ ETDRS letters (6/96) indicates poor visual prognosis as it precludes an eye from treatment with anti-VEGF. ${ }^{15}$ Similarly, a VA of $\leq 20$ ETDRS letters $(3 / 60)$ is the criterion for severe sight impairment registration in the UK.

All data analyses were carried out with R (V.3.5.1). ${ }^{21}$ Calculated means in text and figures are expressed with $\pm \mathrm{SD}$, unless otherwise specified.

\section{Forecasting nAMD patients}

Historical data from 2018 and 2019 for the number of patients initiating anti-VEGF therapy for nAMD by calendar week were used as the basis for a forecast predicting the number of patients needing to initiate anti-VEGF therapy for nAMD during the national lockdown. The number of patients needing to initiate anti-VEGF therapy for nAMD during a period of UK national lockdown between 16 March 2020 and 31 July 2020 (weeks 12 through 31) was forecasted using an additive model consisting of a trend component, a seasonal component and a random component alongside Holt Winters exponential smoothing. ${ }^{22} 23$

\section{Sampling hypothetical nAMD patients based on forecast}

Hypothetical patient profiles were derived for the patients forecasted to require initiation of anti-VEGF therapy for nAMD during the national lockdown. Specifically, hypothetical patients were randomly sampled from patients attending $\mathrm{MEH}$ and UHB during the same time period (16 March to 31 July) in 2018 and 2019.

\section{Propensity scoring and matching of forecasted patients to MARINA trial sham-control patients}

Logistic regression was used to generate a propensity score for each patient in the hypothetical cohort of patients needing to initiate anti-VEGF therapy for nAMD during the national lockdown from the following patient characteristics: age, sex and VA. Using a calliper width of 0.1 of the SD of the logit of the propensity score, the hypothetical patients were matched to corresponding MARINA sham-control arm patients.

\section{Modelling visual outcomes for non-matched patients}

For MEH and UHB patients that could be matched exactly to MARINA patients based on age, sex and VA (matched patients), VA values over time were described using the MARINA 


\begin{tabular}{rcc}
\hline & MEH & UHB \\
\hline $\begin{array}{l}\text { Patients forecasted to initiate anti-VEGF } \\
\text { 16/March - 30/July 2020 }\end{array}$ & 325 & 51 \\
\hline $\begin{array}{l}\text { Historical sample: } \\
\text { Patients who have initiated anti-VEGF treatment }\end{array}$ & \\
16/March - 30/July 2018 & 383 & 46 \\
16/March - 30/July 2019 & 340 & 48 \\
16/March - 30/July 2018 \& 2019 & 723 & 96 \\
\hline
\end{tabular}

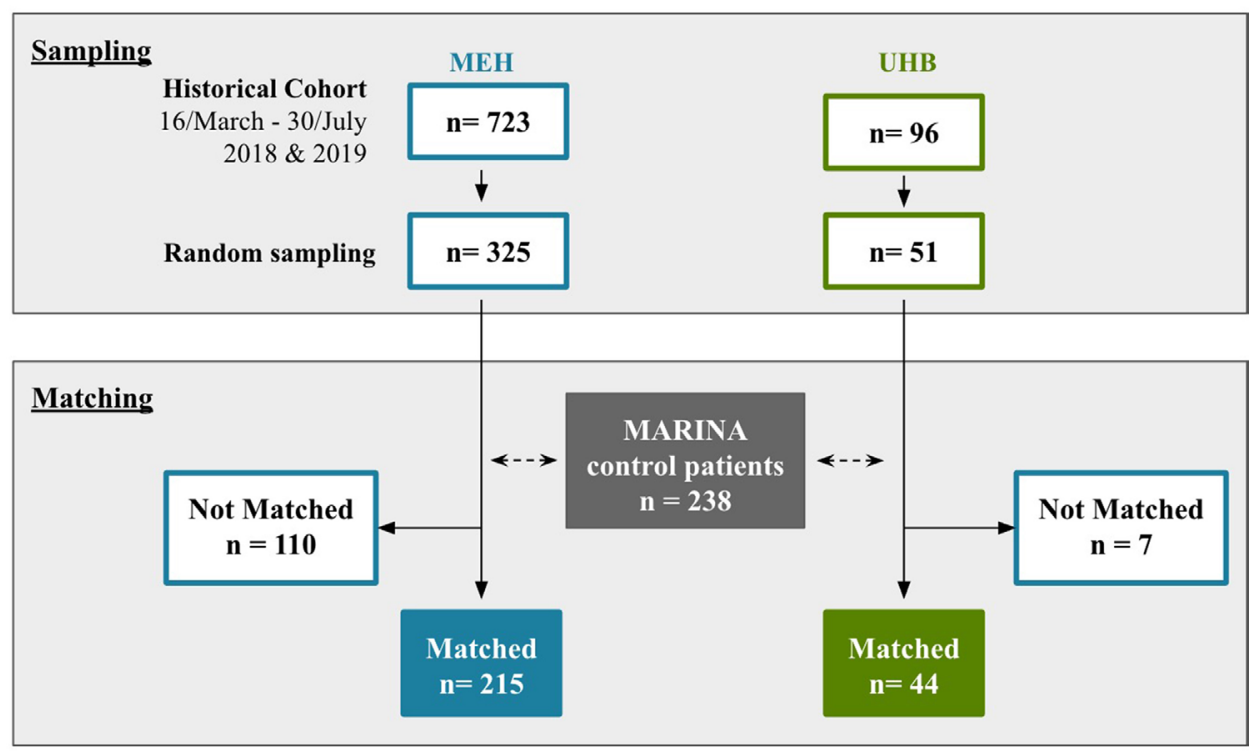

Figure 2 Sampling and matching process to generate cohorts for predicting visual acuity (VA) changes. Flow diagram outlining how patient profiles were generated to model VA changes during a national lockdown. In brief, profiles for the number of neovascular age-related macular degeneration (nAMD) patients forecasted to initiate anti-vascular endothelial growth factor (anti-VEGF) therapy between 16 March 2020 and 31 July 2020 were randomly selected from the pool of patients who initiated therapy in the same period in the two preceding years at Moorfields Eye Hospital (MEH) and University Hospitals Birmingham (UHB). These profiles were matched to patients in the sham-control arm of the Minimally Classic/Occult Trial of the Anti-VEGF Antibody Ranibizumab in the Treatment of Neovascular AMD (MARINA) study on sex, age, VA, VA $\geq 70$ and VA $\leq 20$ at treatment initiation. Some MEH and UHB patients could not be matched to MARINA patient profiles, due to the trial exclusion criteria. Predicted changes in these patients were based on a linear model of VA changes developed from the trial population and extrapolated to the unmatched population. The number of matched and unmatched patients for both NHS Trusts is shown.

sham-control arm matched patient data. For MEH and UHB patients with characteristics out with the MARINA inclusion criteria (non-matched patients), VA was predicted using a simple linear model based on MARINA trial patients with patient characteristics such as age, sex and VA as covariates.

\section{RESULTS}

\section{Cohort characteristics at treatment initiation}

Between 1 January 2018 and 15 March 2020, 1929 treatmentnaïve patients $(60.5 \%$ female $)$ initiated anti-VEGF therapy for nAMD at MEH and 267 patients (68.5\% female) at UHB (table 1A). Most were 65 years or older, with only $6.5 \%$ and $3.0 \%$ of patients under 65 at $\mathrm{MEH}$ and $\mathrm{UHB}$, respectively. The mean VA of eyes initiating anti-VEGF therapy for nAMD between 1 January 2018 and 15 March 2020 at $\mathrm{MEH}$ was $55.9 \pm 16.3$ ETDRS letters (Snellen equivalent 20/80 \pm 1 line), slightly higher than at UHB where it was $51.7 \pm 20.0$ ETDRS letters $(20 / 100 \pm 1$ line (two sample t-test $\mathrm{p}<0.05$; table $1 \mathrm{~B}$ and online supplemental figure 1). Patient cohorts treated at $\mathrm{MEH}$ and UHB were analysed separately given observed differences in demography and clinical features at treatment initiation (table 1).

Forecasting patients needing to initiate anti-VEGF therapy for nAMD during UK national lockdown

Our model to forecast the number of patients needing to initiate anti-VEGF therapy for nAMD during a period of UK national lockdown between 16 March 2020 and 31 July 2020 (weeks 12 through 31) predicted a total of 325 patients in need of initiating anti-VEGF treatment over 20 weeks at MEH (figure 1 and online supplemental table $1 \mathrm{~A})$. At UHB, $51 \mathrm{nAMD}$ patients were forecasted for initiation of anti-VEGF treatment in that period (figure 1 and online supplemental table 1B).

\section{Predicted visual acuity changes in the absence of anti-VEGF therapy}

Next, the impact of absent anti-VEGF treatment due to a national lockdown on vision in nAMD patients was modelled. Here, patients that had presented to MEH and UHB within the 
Table 2 Demography and clinical features at anti-VEGF initiation for the model population

\begin{tabular}{|c|c|c|c|c|c|}
\hline & $\begin{array}{l}\text { MARINA } \\
\text { sham-arm } \\
(\mathrm{n}=238)\end{array}$ & $\begin{array}{l}\text { MEH } \\
\text { matched } \\
(n=215)\end{array}$ & $\begin{array}{l}\text { MEH } \\
\text { not matched } \\
(n=110)\end{array}$ & $\begin{array}{l}\text { UHB } \\
\text { matched } \\
(n=44)\end{array}$ & $\begin{array}{l}\text { UHB } \\
\text { not matched } \\
(n=7)\end{array}$ \\
\hline \multicolumn{6}{|l|}{ Patient demography } \\
\hline \multicolumn{6}{|l|}{$\operatorname{Sex} n(\%)$} \\
\hline Female & $159(66.8)$ & $128(59.5)$ & $73(66.4)$ & $32(72.7)$ & $7(100.0)$ \\
\hline Male & $79(33.2)$ & $87(40.5)$ & 37 (33.6) & $12(27.3)$ & $0(0.0)$ \\
\hline \multicolumn{6}{|l|}{ Age (years) n (\%) } \\
\hline$<65$ & $11(4.6)$ & $9(4.2)$ & $6(5.5)$ & $2(4.5)$ & $0(0.0)$ \\
\hline $65-69$ & $17(7.1)$ & $19(8.8)$ & $4(3.6)$ & $1(2.3)$ & $0(0.0)$ \\
\hline $70-74$ & $50(21.0)$ & $37(17.2)$ & $14(12.7)$ & $4(9.1)$ & $0(0.0)$ \\
\hline $75 \sim 79$ & $68(28.6)$ & $58(27.0)$ & $13(11.8)$ & $4(9.1)$ & $0(0.0)$ \\
\hline $80 \sim 84$ & $64(26.9)$ & $60(27.9)$ & $20(18.2)$ & $19(43.2)$ & $0(0.0)$ \\
\hline $85+$ & $26(10.9)$ & $32(14.9)$ & $53(48.2)$ & $14(31.8)$ & $7(100.0)$ \\
\hline \multicolumn{6}{|l|}{ Ethnicity n (\%) } \\
\hline White & $231(97.1)$ & $97(45.1)$ & $55(50.0)$ & $39(88.6)$ & $6(85.7)$ \\
\hline Not white & $7(2.9)$ & $118(54.9)$ & $55(50.0)$ & $5(11.4)$ & $1(14.3)$ \\
\hline \multicolumn{6}{|l|}{ Smoking status n (\%) } \\
\hline Yes & $130(54.6)$ & $110(51.2)$ & $55(50.0)$ & $33(75.0)$ & $3(42.9)$ \\
\hline No or unknown & $108(45.4)$ & $105(48.8)$ & $55(50.0)$ & $11(25.0)$ & $4(57.1)$ \\
\hline \multicolumn{6}{|l|}{ Clinical features } \\
\hline \multicolumn{6}{|l|}{$\begin{array}{l}\text { Visual acuity (VA) } \\
\text { (ETDRS letters) }\end{array}$} \\
\hline Mean (SD) & $53.61(14.06)$ & $52.92(14.45)$ & $59.50(18.36)$ & $52.55(15.98)$ & $40.57(20.98)$ \\
\hline Median (min, max) & $56.0(3.0,84.0)$ & $55.0(0,86.0)$ & $70.0(11.0,85.0)$ & $55.0(0,76.0)$ & $35.0(11.0,80.0)$ \\
\hline \multicolumn{6}{|c|}{ VA $\geq 70$ ETDRS letters $n(\%)$} \\
\hline Yes & $22(9.2)$ & $16(7.4)$ & $61(55.5)$ & $8(18.2)$ & $1(14.3)$ \\
\hline No & $216(90.8)$ & $199(92.6)$ & $49(44.5)$ & $36(81.8)$ & $6(85.7)$ \\
\hline \multicolumn{6}{|c|}{ VA $\leq 20$ ETDRS letters n (\%) } \\
\hline Yes & $4(1.7)$ & $3(1.4)$ & $3(2.7)$ & $1(2.3)$ & $1(14.3)$ \\
\hline No & $234(98.3)$ & $212(98.6)$ & $107(97.3)$ & $43(97.7)$ & $6(85.7)$ \\
\hline \multicolumn{6}{|c|}{ VA <25 ETDRS letters n (\%) } \\
\hline Yes & $9(3.8)$ & $12(5.6)$ & $7(6.4)$ & $1(2.3)$ & 1 (14.3) \\
\hline No & $229(96.2)$ & $203(94.4)$ & $103(93.6)$ & $43(97.7)$ & $6(85.7)$ \\
\hline
\end{tabular}

Patient characteristics and clinical features for the MARINA sham-control cohort as well as the forecasted patients for the matched and non-matched MEH and UHB cohorts are shown.

MARINA, Minimally Classic/Occult Trial of the Anti-VEGF Antibody Ranibizumab in the Treatment of Neovascular AMD; MEH, Moorfields Eye Hospital; UHB, University Hospitals Birmingham; VEGF, vascular endothelial growth factor.

same 20 -week time period in the 2 years preceding the national lockdown in the UK were randomly selected (figure 2). These patient profiles were matched on demography and clinical features with participants receiving sham-treatments as part of the sham-control arm of the MARINA study, ${ }^{8}$ the last study to include non-VEGF-treated patients.

The resultant cohort comprised 215 matched and 110 unmatched patient profiles for MEH (UHB: 44 matched and 7 unmatched). Table 2 summarises the presenting characteristics for the patients in the MARINA sham arm, as well as, matched and unmatched MEH and UHB cohorts. A smaller proportion of $\mathrm{MEH}$ patients matched to patient profiles in the sham-control arm of the MARINA trial when compared with UHB.

Our data suggest that an interruption to ophthalmic services during a national lockdown period would lead to VA deterioration in new nAMD patients (online supplemental figure 2). If left untreated for 12 months, mean VA was projected to

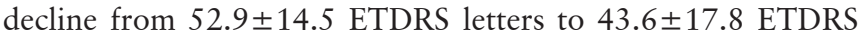
letters in the MEH-MARINA matched cohort, and from 52.6 \pm 16.0 ETDRS letters to 40.6 \pm 17.0 ETDRS letters in the UHB-MARINA matched cohort (online supplemental figure 2 and online supplemental table 2). For unmatched patients, VA changes were extrapolated using a separate model, also based on the data from the MARINA sham-control cohort. For the $\mathrm{MEH}$ and UHB non-matched patients, mean VA after 12 months was projected to decline from 59.5 \pm 18.4 to $41.9 \pm 12.3$ ETDRS letters and from $40.6 \pm 21.0$ to $29.5 \pm 8.2$ ETDRS letters $(n=7)$, respectively (online supplemental table 2). A decline in VA was consistently seen as early as 3 months following failure to initiate treatment.

\section{Impact on patient quality of life in untreated nAMD due to national lockdown}

Forecasted changes in VA observed here are likely to be clinically significant. At baseline, 25\% (83/325) of $\mathrm{MEH}$ patients, forecasted to require anti-VEGF, would be expected to have a VA $\geq 70$ ETDRS letters $(6 / 12)$ in the affected eye, based on a pooled analysis of matched and unmatched cohorts. The pooled UHB cohort was predicted to have a lower VA at baseline where only $9.8 \%$ (5/51) of patients with VA $\geq 70$ ETDRS letters (6/12). After 3 months without treatment, this proportion was projected 

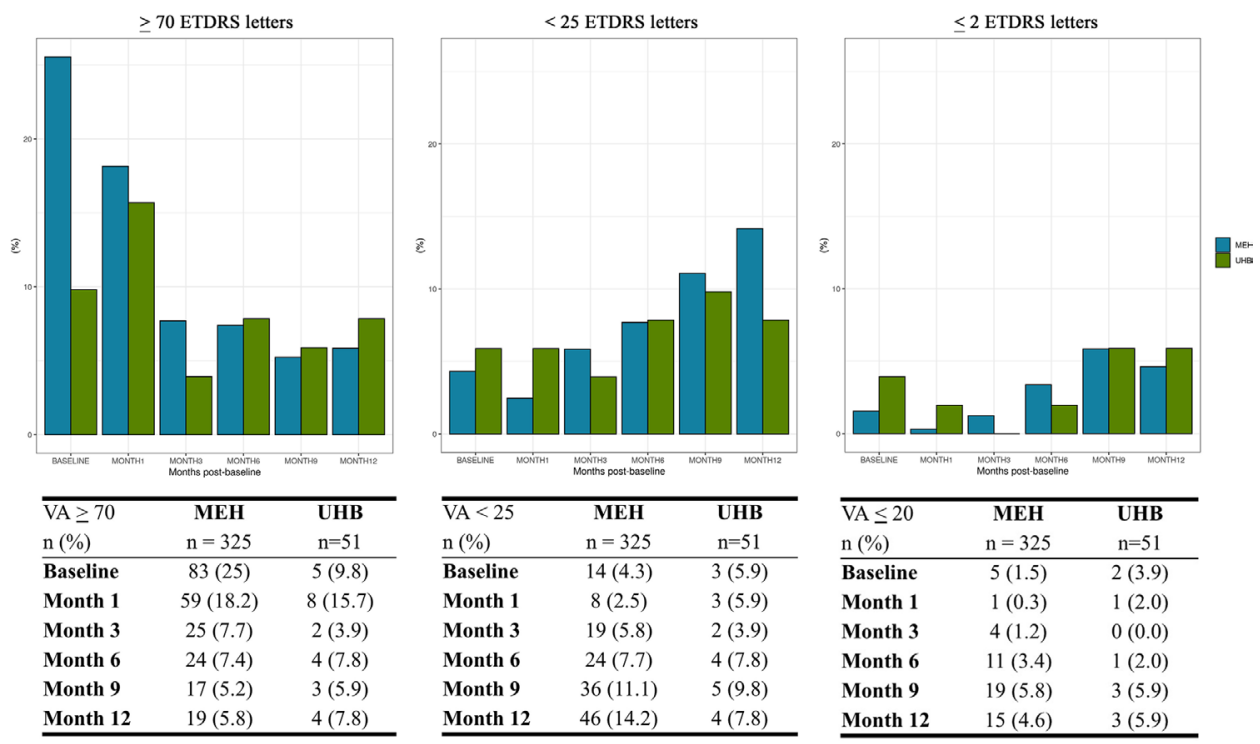

Figure 3 Projected visual acuity (VA) thresholds. Bar charts representing the proportion of study eyes for the Moorfields Eye Hospital (MEH) (blue) and University Hospitals Birmingham (UHB) (green) predicted cohorts over time to (A) achieve a VA of $\geq 70$ ETDRS, (B) fall below a VA of 25 ETDRS letters and (C) have a VA of $\leq 20$ ETDRS letters. Matched and unmatched cohorts were combined for this analysis. Last observation was carried over to keep constant denominators.

to fall to $7.7 \%(25 / 325)$ and $3.9 \%(2 / 51)$ for $\mathrm{MEH}$ and UHB, respectively. After 12 months without treatment, the proportion of eyes with VA $\geq 70$ ETDRS letters (6/12) was projected to be $5.8 \%(19 / 325)$ and $7.8 \%(4 / 51)$ for $\mathrm{MEH}$ and $\mathrm{UHB}$, respectively (figure $3 \mathrm{~A}$ ).

For those forecasted to initiate anti-VEGF in the national lockdown period, $14.2 \%(46 / 325)$ and $7.8 \%(4 / 51)$ of study eyes at $\mathrm{MEH}$ and UHB, respectively, would not be eligible for treatment (ie, VA $<25$ ETDRS letters (6/96)) at the 12-month time point without treatment (figure $3 \mathrm{~B}$ ).

Of forecasted nAMD patients, 1.5\% (5/325) and 3.9\% (2/51) at $\mathrm{MEH}$ and $\mathrm{UHB}$ cohorts, respectively, were predicted to have a VA of $\leq 20$ ETDRS letters (3/60) at baseline. After 12 months delay in treatment, proportion falling below this threshold is projected to increase to $4.6 \%(15 / 325)$ and 5.9\% (3/51) at $\mathrm{MEH}$ and $\mathrm{UHB}$, respectively (figure $3 \mathrm{C}$ ).

\section{DISCUSSION}

\section{Main findings}

Based on data between 2018 and 2019, 376 patients were projected to initiate anti-VEGF therapy for nAMD across $\mathrm{MEH}$ and UHB between 16 March 2020 and 31 July 2020. In the absence of anti-VEGF therapy, these patients would have-on average-lost vision. Analyses presented here suggest that a treatment-naïve nAMD patient presenting at the beginning of service disruption (end of April) would have had a reduced chance of retaining good vision (VA $\geq 70$ ETDRS letters $(6 / 12)$ ) once the services resumed at the end of July 2020. Crucially, treatment-delay may also have put them at risk of losing eligibility for treatment, as they may no longer have met the minimum VA requirement of $\geq 25$ ETDRS letters (6/96), in addition to increased risk of severe visual impairment $(\leq 20$ ETDRS letters $(3 / 60))$. Risks to positive visual outcomes would proportionately increase if service disruption lasted longer than 6 months, either due to: extended lockdown period; reduced capacity on service resumption or if patients did not feel safe to seek medical services.

\section{Results in the context of the existing literature}

Anti-VEGF is used to treat VA deterioration secondary to nAMD. ${ }^{8} 2425$ Interruption to therapy can negatively impact visual prognosis ${ }^{26}$ and it has been demonstrated that early treatment initiation results in better prognosis for patients with nAMD. Indeed, time-event analyses of $\mathrm{MEH}$ nAMD patients demonstrated that probability of attaining 70 ETDRS letters $(6 / 12)$ increases by $43 \%$ for every 5 letters of VA at treatment initiation. ${ }^{27}$ Moreover, a higher baseline VA also increases the duration of retaining positive visual outcome; and treatment delay can have a detrimental effect on vision outcomes.

The vision recovery velocity for anti-VEGF therapy was estimated to be between 0.17 and 0.56 letters per month. ${ }^{28}$ Analyses presented here demonstrated that vision loss could proceed as fast as one letter per month of deferred treatment, that is, at a faster rate than can be regained on a monthly basis with the gold standard treatment. This illustrates the importance of timely treatment initiation for optimising vision outcomes.

The effects of immediate disruption of medical services due to national lockdown is becoming evident, both within AMD pathways, ${ }^{29}$ ophthalmic services ${ }^{30}$ and more broadly across each medical specialty. ${ }^{31}$ Single-centre studies have reported reduction in numbers of newly diagnosed nAMD presenting for treatment between prepandemic and postlockdown time frames, acknowledging the difficulty in predicting treatment outcomes in those for whom treatment was delayed or not initiated. ${ }^{29}$ Retina specialists anticipated potential detrimental effects of delayed diagnosis and interrupted care, and identified ways to mitigate this with accelerated innovation, particularly in healthcare pathway redesign and digitalisation. ${ }^{32}$

\section{Limitations}

Our predictive model for VA over time is based on post hoc analysis of the landmark MARINA trial sham-control arm, which excludes patients with any concurrent intraocular condition in the study eye (eg, cataract or diabetic retinopathy). In the wider population, concurrent other conditions that could impair VA 
and visual outcomes over time need to be accounted for, and therefore the model may underestimate the loss of VA over time.

A proportion of MEH and UHB patients could not be matched to MARINA patient profiles, due to differences in patient selection for inclusion in the respective cohorts. For example, the majority of patients predicted to fall below 70 ETDRS letters (6/12) are based on patients who did not match the MARINA trial population due to its selection criteria. Predicted changes in these patients were based on a linear model of VA changes developed from the trial population and extrapolated to the unmatched population. Our analyses suggest worse visual outcomes for non-matched patients, especially for the MEH cohort. This effect could reflect the higher mean starting VA in the MEH cohort compared with UHB and MARINA cohorts.

There are two anti-VEGF treatments licensed in the UK, ranibizumab and aflibercept. Aflibercept is most commonly used at $\mathrm{MEH}$, in contrast to ranibizumab at UHB. While both treatments inhibit VEGF signalling in nAMD, any influence of treatment choice on visual outcomes could not be addressed in the present study.

\section{Implications for further research and practice}

This study demonstrates the value of coordinated health data research initiatives such as INSIGHT. Furthermore, insights from prospectively collected routine clinical patient-level hospital data can be maximised by combining them with additional resources, such as randomised clinical trial data. Going forward, the INSIGHT initiative would also enable validation of our model with observed data collected during the first lockdown, as well as model the effects of subsequent lockdowns in the UK. Such initiatives could help the medical community better understand and potentially mitigate the effects of national lockdowns on other chronic health conditions where early treatment initiation is critical for patient outcomes.

\section{CONCLUSION}

As COVID-19 cases decrease, hospitals seek to progressively restore non-urgent services in the context of competing demands for limited resources. According to our results, a 3-month delay in treatment initiation could potentially result in measurable detrimental effects on vision. Extending the delay for longer periods of time is predicted to proportionately decrease positive visual outcomes and increase negative ones. It is now essential to support patients accessing care by providing safe environments as recommended by national bodies. ${ }^{33}$ Providing optimal patient care, while minimising the risk of infection, resource planning at ophthalmic care centres must take into account the bolus of patients who were undiagnosed or did not initiate therapy during planned lockdowns. This group of patients should be prioritised and resources allocated appropriately to diagnose and treat these patients as they present for care.

This INSIGHT Health Data Research Hub report has modelled the immediate impact of delayed diagnosis of nAMD and estimated the potential of permanent visual morbidity caused by anti-VEGF treatment interruptions secondary to national lockdown. The directed use of MARINA trial data enabled a forecast of this serious morbidity.

\section{Twitter Dun Jack Fu @dunjackfu and Wen Hwa Lee @againstAMD}

Acknowledgements We would like to acknowledge the following people for their time and dedication: to the INSIGHT Health Data Research Hub Dr Helen Baker, Public and Patient Involvement (PPI) Lead of INSIGHT and PPI Lead, NIHR Biomedical Research Centre at Moorfields Eye Hospital NHS Foundation Trust and UCL Institute of Ophthalmology, London, UK; and Helen Khan, Communications Lead for INSIGHT,
Communications Manager, NIHR Biomedical Research Centre at Moorfields Eye Hospital NHS Foundation Trust and UCL Institute of Ophthalmology, London, UK; Manijt Benning, Chief Operating Officer, Moorfields Eye Hospital NHS Foundation Trust, London, UK; Hilary Fanning, INSIGHT Managing Director and Director of Research Development and Innovation, University Hospitals Birmingham NHS Foundation Trust, Birmingham, UK; to key contributors from Roche and Genentech Jessica Davies, Anh Duong, Claudia Felix, Manoj Hampiholi, Bikash Sabata, Mani Pollachi Subramanyam, Eric Wang and to the MARINA participants, investigators and study team.

Contributors SPM: conception, study design, data acquisition and analysis, manuscript preparation. She is guarantor. AKD: conception, study design, data acquisition, manuscript revision. PAK: conception, study design, data acquisition and analysis, manuscript revision. RC: conception, study design, statistical analysis plan, data acquisition and analysis, manuscript preparation. DJF: analysed the data, manuscript preparation. C-YC: analysed the data and revised the manuscript. JGG, $\mathrm{GHL}, \mathrm{JJH}$ and $\mathrm{CH}$ : conception and revised the manuscript. All authors approved the final paper for submission and publication.

Funding INSIGHT is a National Health Service-led partnership established to improve healthcare by encouraging research using routinely collected eye data. INSIGHT is funded by Health Data Research, UKnited Kingdom (HDR UK). HDR UK is funded by the Medical Research Council (MRC), Engineering and Physical Sciences Research Council (EPSRC), Economic and Social Research Council (ESRC), National Institute for Health Research (NIHR); Chief Scientist Office of the Scottish Government Health and Social Care Directorates, Health and Social Care Research and Development Division (Welsh Government; HCRW), Public Health Agency (Northern Ireland; PHA), British Heart Foundation (BHF) and the Wellcome Trust Limited as trustee of the Wellcome Trust. PAK has received speaker fees from Heidelberg Engineering, Topcon, Carl Zeiss Meditec, Haag-Streit, Allergan, Novartis and Bayer. He has served on advisory boards for Novartis and Bayer and has been an external consultant for DeepMind and Optos. DJF reports other financial activities outside the submitted work with Allergan. SPM reports speaker fees from Allergan, Chugai-Roche Ltd, Chiesi, Heidelberg Engineering, Novartis, Roche, Santen, Santhera and Teva. She has served on advisory boards for Invex Therapeutics, Janssen and Roche. She is an external consultant for Neurodiem, outside the submitted work. RC: employee, Genentech and stock Roche. CC: employee, Genentech and stock Roche JGG: employee, Genentech and stock Roche. CH: employee, Google Health UK and stock Alphabet.

\section{Competing interests None declared.}

\section{Patient consent for publication Not required.}

Ethics approval The study was conducted in compliance with the tenets of the Declaration of Helsinki and has approval from the relevant Institutional Review Board and ethics committee (West Scotland REC 4 (20/WS/0087)).

Provenance and peer review Not commissioned; externally peer reviewed.

Data availability statement Data may be obtained from a third party and are not publicly available.

Supplemental material This content has been supplied by the author(s). It has not been vetted by BMJ Publishing Group Limited (BMJ) and may not have been peer-reviewed. Any opinions or recommendations discussed are solely those of the author(s) and are not endorsed by BMJ. BMJ disclaims all liability and responsibility arising from any reliance placed on the content. Where the content includes any translated material, BMJ does not warrant the accuracy and reliability of the translations (including but not limited to local regulations, clinical guidelines, terminology, drug names and drug dosages), and is not responsible for any error and/or omissions arising from translation and adaptation or otherwise.

Open access This is an open access article distributed in accordance with the Creative Commons Attribution Non Commercial (CC BY-NC 4.0) license, which permits others to distribute, remix, adapt, build upon this work non-commercially, and license their derivative works on different terms, provided the original work is properly cited, appropriate credit is given, any changes made indicated, and the use is non-commercial. See: http://creativecommons.org/licenses/by-nc/4.0/.

\section{ORCID iDs}

Dun Jack Fu http://orcid.org/0000-0003-2852-6912

Alastair K Denniston http://orcid.org/0000-0001-7849-0087

\section{REFERENCES}

1 Bressler NM. Age-Related macular degeneration is the leading cause of blindness. JAMA 2004;291:1900-1.

2 Jager RD, Mieler WF, Miller JW. Age-related macular degeneration. N Eng/ J Med 2008;358:2606-17.

3 Owen CG, Fletcher AE, Donoghue $M$, et al. How big is the burden of visual loss caused by age related macular degeneration in the United Kingdom? Br J Ophthalmol 2003;87:312-7. 
4 Wood JM, Lacherez P, Black AA, et al. Risk of falls, injurious falls, and other injuries resulting from visual impairment among older adults with age-related macular degeneration. Invest Ophthalmo/ Vis Sci 2011;52:5088-92.

5 Lotery A, Xu X, Zlatava G, et al. Burden of illness, visual impairment and health resource utilisation of patients with neovascular age-related macular degeneration: results from the UK cohort of a five-country cross-sectional study. Br J Ophthalmol 2007;91:1303-7.

6 Cox TM, ffytche DH. Negative outcome charles bonnet syndrome. $\mathrm{Br} J$ Ophthalmol 2014;98:1236-9.

7 Owen CG, Jarrar Z, Wormald R, et al. The estimated prevalence and incidence of late stage age related macular degeneration in the UK. Br J Ophthalmol 2012;96:752-6.

8 Rosenfeld PJ, Brown DM, Heier JS, et al. Ranibizumab for neovascular age-related macular degeneration. N Engl J Med 2006;355:1419-31.

9 Heier JS, Brown DM, Chong V, et al. Intravitreal aflibercept (VEGF trap-eye) in wet age-related macular degeneration. Ophthalmology 2012;119:2537-48.

10 Solomon SD, Lindsley K, Vedula SS, et al. Anti-vascular endothelial growth factor for neovascular age-related macular degeneration. Cochrane Database Syst Rev 2019;2019.

11 The Royal College of Ophthalmologists. RCOphth: management of ophthalmology services during the Covid pandemic, 2020. Available: https:/www.rcophth.ac.uk/wpcontent/uploads/2020/03/RCOphth-Management-of-Ophth [Accessed 13 May 2020]

12 Moorfields Eye Hospital NHS Foundation Trust. Moorfields eye hospital nhs foundation trust ophthalmological risk stratification \& implementation guidance v2.0, 2020. Available: https://www.rcophth.ac.uk/wp-content/uploads/2020/03/MoorfieldsOphthalmic-Ris [Accessed 13 May 2020].

13 NHS. National data opt-out operational policy guidance document v4.0, 2020 Available: https://digital.nhs.uk/services/national-data-opt-out/operational-policyguidan [Accessed 04 Aug 2020].

14 Thomas DS, Warwick A, Olvera-Barrios A. Estimating excess visual loss in people with neovascular age-related macular degeneration during the COVID-19 pandemic. MedRxiv 2020 https://www.medrxiv.org/content/

15 National institute for health and care excellence. Age-related macular degeneration NICE guideline [NG82], 2018. Available: https://www.nice.org.uk/guidance/ng82/ chapter/Recommendations [Accessed 08 Jan 2021].

16 Gregori NZ, Feuer W, Rosenfeld PJ. Novel method for analyzing snellen visual acuity measurements. Retina 2010;30:1046-50

17 Fontenot JL, Bona MD, Kaleem MA, Olsen TW, Harris J, Mizuiri D, et al. Vision rehabilitation preferred practice pattern®. Ophthalmology 2018;125:P228-78.

18 Visual standards: aspects and ranges of vision loss with emphasis on population surveys : report prepared for the international council of ophthalmology at the 29th international congress of ophthalmology, 2002. International Council of ophthalmology. Available: http://www.icoph.org/resources/10/Visual-Standards--Aspects-and-Ranges-of-Vision-Loss.html
19 Bron AM, Viswanathan AC, Thelen U, et al. International vision requirements for driver licensing and disability pensions: using a milestone approach in characterization of progressive eye disease. Clin Ophthalmol 2010;4:1361-9.

20 Lamoureux EL, Hassell JB, Keeffe JE. The impact of diabetic retinopathy on participation in daily living. Arch Ophthalmol 2004;122:84-8.

21 Team R. Rstudio: Integrated development environment for $r$ [Computer software manual]. Boston, MA, 2016.

22 Holt CC. Forecasting trends and seasonals by exponentially weighted moving averages, ONR research memorandum, carnegie Institute of technology 52. PR Winters (1960) forecasting sales by exponentially weighted moving averages. Manage Sci 1957;6:324-42

23 Winters PR. Forecasting sales by exponentially weighted moving averages. Manage Sci 1960;6:324-42.

24 Patel PJ, Bunce $C$, Tufail $A$, et al. A randomised, double-masked phase III/IV study of the efficacy and safety of avastin(R) (Bevacizumab) intravitreal injections compared to standard therapy in subjects with choroidal neovascularisation secondary to age-related macular degeneration: clinical trial design. Trials 2008;9:56.

25 Dugel PU, Koh A, Ogura Y, et al. Hawk and harrier: phase 3, multicenter, randomized, double-masked trials of Brolucizumab for neovascular age-related macular degeneration. Ophthalmology 2020;127:72-84.

$26 \mathrm{Kim} \mathrm{JH}$, Chang YS, Kim JW. Natural course of patients discontinuing treatment for age-related macular degeneration and factors associated with visual prognosis. Retina 2017:37:2254-61.

27 Fu DJ, Keenan TD, Faes L, et al. Insights from survival analyses during 12 years of anti-vascular endothelial growth factor therapy for neovascular age-related macular degeneration. JAMA Ophthalmol 2021;139:57-67.

28 Almeida DRP, Ruzicki J, Xu K, et al. Vision recovery velocity, momentum and acceleration: advanced vitreoretinal analytics as measure of treatment efficacy for neovascular age-related macular degeneration. Clin Ophthalmol 2021;15:189-94.

29 Yang K-B, Feng $\mathrm{H}$, Zhang $\mathrm{H}$. Effects of the COVID-19 pandemic on anti-vascular endothelial growth factor treatment in China. Front Med 2020;7:576275.

30 Thaller M, Tsermoulas G, Sun R, et al. Negative impact of COVID-19 lockdown on papilloedema and idiopathic intracranial hypertension. J Neurol Neurosurg Psychiatry 2021;92:795-7.

31 Ball S, Banerjee A, Berry C, et al. Monitoring indirect impact of COVID-19 pandemic on services for cardiovascular diseases in the UK. Heart 2020;106:1890-7.

32 Sim SS, Yip MY, Wang Z, et al. Digital technology for AMD management in the postCOVID-19 new normal. Asia Pac J Ophthalmol 2021;10:39-48.

33 American Society of Retina Specialists. COVID-19: patient update. Available: https:// www.asrs.org/content/documents/covid-19-patient-update-v5.pdf [Accessed 08 Jan 2021]. 